\title{
Influence of water-methanol solution additives on hydrocarbon fuel combustion in burner
}

\author{
Aleksandr S. Naumkin ${ }^{1, *}$, Boris V. Borisov ${ }^{1}$, and Aleksandr G. Nigay ${ }^{1}$ \\ ${ }^{1}$ National Research Tomsk Polytechnic University, 634050 Tomsk, Russia
}

\begin{abstract}
Mathematical model of burning disposal a water-methanol solution by the gas fuel is presented. Two-dimensional model in the ANSYS Fluent Software is made. Thermal distribution of the flare and distribution of methane concentration are derived. We analysed the results of mathematical modelling.
\end{abstract}

\section{Introduction}

Ecological aspects of human life are becoming more important every year. Disposal of waste without harm to the environment is costly; therefore, the economic efficiency in choosing methods of utilization is tFhe important factor for the any industry [1].

In this paper, we consider the disposal of the water-methanol solution. This solution is a waste of the technological cycle of extraction and preparation for transportation by pipeline transport of natural gas. Most of the methanol is separated in the distillation column, but the residue contains a small volume of methanol in water, which is harmful to the environment. Currently, disposal of water-methanol solution is produced by incineration. About 60 commercial companies in Russia use the GFU-5.

\section{Research method and data}

The processes accompanying disposal by the example of burning a water-methanol solution is simulated. This is the basis for developing a methodology for calculating the considerate process. A water-methanol solution is the following volumetric composition: H2O-99.67\%, $\mathrm{CH} 3 \mathrm{OH}-0.33$ and the fuel is a natural gas of the following volumetric composition: $\mathrm{CH} 4-$ 98.9\%; C2H6-0.12\%; C3H8-0.01\%; C4H10-0.01\%; CO2-0.06\%; N2-0.9\%.

LLC “TyumenNIIgiprogaz" has developed GFU-5 which shown in Fig. 1 [2].

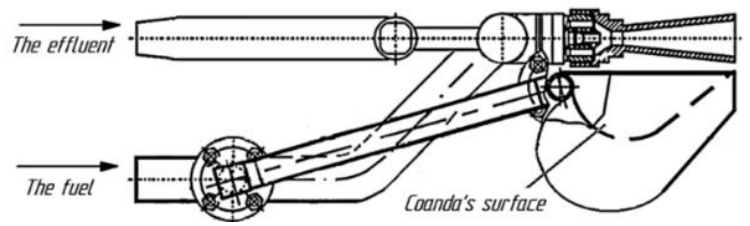

Fig. 1. Flare facility of GFU-5.

\footnotetext{
*Corresponding author: alexnaumserg93@mail.ru
} 
Fuel gas consumption for atomization of industrial effluents is $0.1478 \mathrm{~m}^{3} / \mathrm{s}$. Flow rate of combustible gas to Coanda's surface (Fig. 1) is $0.0739 \mathrm{~m}^{3} / \mathrm{s}$. Consumption of water watermethanol is $0.278 \mathrm{~kg} / \mathrm{s}$. $55 \mu \mathrm{m}$.

The dimensions of the droplets formed at a given ratio of the gas and liquid phases were

The stationary model of the flame propagation in a two-dimensional setting is consider. Part of the fuel, aimed at spraying industrial effluents, moves inside the cylindrical pipeline. The industrial effluents comes from a coaxially located pipeline, smaller diameter, into the fuel flow through the nozzle.

The stationary model is solved by the establishment method. The process of mixing different phases is described by a system of differential equations. The system contains the equations of mass, momentum and energy conservation [3]:

$$
\left\{\begin{array}{c}
\frac{\partial \rho}{\partial t}+\nabla(\rho \vec{v})=S_{m} \\
\frac{\partial}{\partial t}(\rho \vec{v})+\nabla(\rho \vec{v} \vec{v})=-\nabla p+\nabla(\overline{\bar{\tau}})+\rho \vec{g}+\vec{F} \\
\frac{\partial}{\partial t}(\rho e)+\nabla[\vec{v}(\rho e+p)]=-\nabla\left(\sum_{i} h_{i} J_{i}\right)+S_{h}
\end{array}\right.
$$

where $\overline{\bar{\tau}}=\mu\left[\left(\nabla \vec{v}+\nabla \vec{v}^{T}\right)-2 / 3 \nabla \vec{v} I\right]-$ viscous stress tensor; $I-$ unit tensor; $\mu-\mathrm{dy}-$ namic viscosity, $\mathrm{Pa} \cdot \mathrm{s} ; t$ - time, $\mathrm{s} ; \rho$ - density, $\mathrm{kg} / \mathrm{m}^{3} ; \vec{v}$ - velocity vector, $\mathrm{m} / \mathrm{s} ; J$ - diffusion current density, $\mathrm{kg} /\left(\mathrm{m}^{2} \cdot \mathrm{s}\right) ; F-$ external force, $\mathrm{N} ; g-$ gravitational acceleration, $\mathrm{m} / \mathrm{s}^{2} ; p-$ static pressure, $\mathrm{Pa} ; \mathrm{e}=h-p / \rho+v^{2} / 2-$ specific total energy, $\mathrm{J} / \mathrm{kg} ; h=\sum_{i} h_{i} J_{i}-$ specific total enthalpy, $\mathrm{J} / \mathrm{kg} ; h_{i}=\int_{T_{r e f}}^{T} c_{p, i} d T-$ specific total enthalpy of component $i, \mathrm{~J} / \mathrm{kg}$; $T_{\text {ref }}=298.15$ - reference temperature, $\mathrm{K} ; c_{p, i}$ - heat capacity at constant pressure of component $i, \mathrm{~J} /(\mathrm{K} \cdot \mathrm{kg}) ; S_{m}=\sum_{i}^{N}\left(Y_{i} S_{i}\right)-$ source of mass, $\mathrm{kg} /\left(\mathrm{m}^{3} \cdot \mathrm{s}\right) ; S_{h}=-\sum_{i} h_{i}^{o} R_{i} / M_{j}-$ the volumetric heat sources of the chemical reactions, $\mathrm{J} /\left(\mathrm{m}^{3} \cdot \mathrm{s}\right) ; R_{i}$ - the net source of chemical species i due to reaction, $\mathrm{mol} /\left(\mathrm{m}^{3} \cdot \mathrm{s}\right) ; h_{i}^{0}-$ standard state enthalpy, $\mathrm{J} / \mathrm{mol} ; M_{i}-$ mole volume of components $i, \mathrm{~m}^{3} / \mathrm{mol}$.

Part of the gaseous fuel that passes through the nozzle forms a gas-droplet mixture with a water-methanol solution. Another part of the gaseous fuel flowing around the Coanda's surface comes to the periphery of the torch, where there is a mutual diffusion of the two streams and the environmental air to form a fuel mixture.

We model the diffusion process of a multicomponent mixture in accordance with the Maxwell-Stefan equations. Diffusion pressure is low [4]:

$$
\sum_{\substack{j=1 \\ j \neq i}}^{N} \frac{X_{i} X_{j}}{D_{i j}}\left(\vec{V}_{J}-\vec{V}_{l}\right)=d_{i}-\frac{\nabla T}{T} \sum_{\substack{j=1 \\ j \neq i}}^{N} \frac{X_{i} X_{j}}{D_{i j}}\left(\frac{D_{T, j}}{\rho_{j}}-\frac{D_{T, i}}{\rho_{i}}\right)
$$

where $X_{i}$ - mole fraction of component $\mathrm{i} ; V_{i}$ - diffusion velocity of component $\mathrm{i}, \mathrm{m}^{2} / \mathrm{s}$; $D_{i j}$ - binary mass diffusion coefficient, $\mathrm{m}^{2} / \mathrm{s} ; D_{T}-$ thermal diffusion coefficient, $\mathrm{m}^{2} / \mathrm{s} ; T-$ temperature, $\mathrm{K} ; d_{i}$ - particle diameter, $\mathrm{m}$.

The Maxwell diffusion coefficient is considered equal to the binary diffusion coefficient for an ideal gas. The barodiffusion effect, excluding the gravitational effect, is written by the following expression:

$$
\overrightarrow{J_{l}}=\rho_{i} \vec{V}_{l}
$$




$$
\begin{gathered}
d_{i}=\nabla X_{i}, \\
\sum_{\substack{j=1 \\
j \neq i}}^{N} \frac{X_{i} X_{j}}{D_{i j}}\left(\frac{\overrightarrow{J_{j}}}{\rho_{j}}-\frac{\overrightarrow{J_{l}}}{\rho_{i}}\right)=\nabla X_{i}-\frac{\nabla T}{T} \sum_{\substack{j=1 \\
j \neq i}}^{N} \frac{X_{i} X_{j}}{D_{i j}}\left(\frac{D_{T, j}}{\rho_{j}}-\frac{D_{T, i}}{\rho_{i}}\right)
\end{gathered}
$$

In order to find the diffusion flux of a chemical species use Fick's law:

$$
\overrightarrow{J_{l}}=-\sum_{j=1}^{N-1}\left(\rho D_{i, m}+\frac{\mu_{t}}{S c_{t}}\right) \nabla Y_{i}-D_{T, i} \frac{\nabla T}{T}
$$

where $Y_{i}$ - the mass fraction of species $i$; $S c_{t}$ - turbulent Schmidt number; $\mu_{t}=$ $\rho C_{\mu} k^{2} / \epsilon$ - eddy viscosity, $\mathrm{Pa} \cdot \mathrm{s} ; C_{\mu}=0.09$ - constant [5]; $\epsilon$ - dissipation rate, $\mathrm{m}^{2} / \mathrm{s}^{3} ; k-$ eddy kinetic energy, $\mathrm{m}^{2} / \mathrm{s}^{2} ; D_{i, m}=\left(1-X_{i}\right) / \sum_{i j \neq i}\left(X_{i} / D_{i j}\right)$ - the mass diffusion coefficient for species $i, \mathrm{~m}^{2} / \mathrm{s}$.

The equation of the diffusion flux vector of the mass component through the generalized diffusion coefficients $A$ and $B$ is consider. The following expression determin the diffusion coefficient of the component $i$ in $j$ :

$$
D_{i j}=[D]=[A]^{-1}[B] .
$$

In the matrix of generalized diffusion coefficients according to the Fick's law, the coefficients $\mathrm{A}$ and $\mathrm{B}$ are determined by the following expression, according to [6]:

$$
\begin{gathered}
A_{i i}=-\left(\frac{X_{i}}{D_{i N}} \frac{M_{w}}{M_{w, N}}+\sum_{\substack{i=1 \\
j \neq 1}}^{N} \frac{X_{i}}{D_{i j}} \frac{M_{w}}{M_{w, i}}\right), \\
A_{i j}=X_{i}\left(\frac{1}{D_{i j}} \frac{M_{w}}{M_{w, j}}-\frac{1}{D_{i N}} \frac{M_{w}}{M_{w, N}}\right), \\
B_{i i}=-\left(X_{i} \frac{M_{w}}{M_{w, j}}+\left(1-X_{i}\right) \frac{M_{w}}{M_{w, i}}\right), \\
B_{i j}=X_{i}\left(\frac{M_{w}}{M_{w, j}}-\frac{M_{w}}{M_{w, N}}\right)
\end{gathered}
$$

where $M_{w}$ - the molecular weight, $\mathrm{kg} / \mathrm{kmol}$.

The coefficients of thermal diffusion is determined in accordance to [7] by the following expression:

$$
D_{T, i}=-2.59 \times 10^{-7} T^{0.659}\left[\frac{M_{w, i}^{0.511} X_{i}}{\sum_{i=1}^{N} M_{w, i}^{0.511} X_{i}}-Y_{i}\right] \cdot\left[\frac{\sum_{i=1}^{N} M_{w, i}^{0.511} X_{i}}{\sum_{i=1}^{N} M_{w, i}^{0.489} X_{i}}\right],
$$

The turbulence effects are determined in according by standard $k-\epsilon$ model. This model are includes the transport of turbulent kinetic energy and the dissipation rate of turbulent kinetic energy equations [8]:

$$
\frac{\partial}{\partial t}(\rho k)+\frac{\partial}{\partial x_{j}}\left(\rho k u_{i}\right)=\frac{\partial}{\partial x_{j}}\left[\left(\mu+\frac{\mu_{t}}{\sigma_{k}}\right) \frac{\partial k}{\partial x_{j}}\right]+G_{k}-\rho \epsilon,
$$




$$
\frac{\partial}{\partial t}(\rho \epsilon)+\frac{\partial}{\partial x_{j}}\left(\rho \epsilon u_{i}\right)=\frac{\partial}{\partial x_{j}}\left[\left(\mu+\frac{\mu_{t}}{\sigma_{\epsilon}}\right) \frac{\partial \epsilon}{\partial x_{j}}\right]+C_{1 \epsilon} \frac{\epsilon}{k} C_{k}-C_{2 \epsilon} \rho \frac{\epsilon^{2}}{k},
$$

where $G_{k}=-\rho \overline{u_{l}^{\prime} u_{j}^{\prime}} \partial u_{j} / \partial x_{j}$ - the production of turbulence kinetic energy, $\mathrm{kg} /\left(\mathrm{m} \cdot \mathrm{s}^{3}\right) ; k$ - turbulence kinetic energy, $\mathrm{m}^{2} / \mathrm{s}^{2} ; \epsilon$ - turbulent dissipation rate, $\mathrm{m}^{2} / \mathrm{s}^{3} ; C_{1 \epsilon}=1.44$ - empirical constant; $C_{2 \epsilon}=1.92-$ empirical constant; $C_{\mu}=0.09$ - empirical constant; $\sigma_{k}=1.0-$ empirical constant; $\sigma_{\epsilon}=1.3$ - empirical constant [5].

The chemical reactions are simulated in according by the mass conservation law of components i.

$$
\frac{\partial \rho Y_{i}}{\partial t}+\nabla\left(\rho \vec{v} Y_{i}\right)=-\nabla \overrightarrow{J_{l}}+R_{i}+S_{i}
$$

where $R_{i}$ - the net rate of production of species $i$ by chemical reaction, $\mathrm{kg} /\left(\mathrm{s} \cdot \mathrm{m}^{3}\right) ; S_{i}-$ rate of creation by addition from the dispersed phase, $\mathrm{kg} /\left(\mathrm{s} \cdot \mathrm{m}^{3}\right) ; Y_{i}-$ the mass fraction of species $i$.

The combustion model from a single gross reaction for each fuel component is considered. The reaction rate is calculated in according by the vortex dissipation model. Turbulent mixing affects the rate of chemical reactions. The lower value from the speed of the diffusion process and the rate of chemical reactions in according by the Arrhenius law is taken to determine the reaction rate [10].

$$
\begin{gathered}
R_{i, r}=v_{i, r}^{\prime} M_{w, i} A \rho \frac{\epsilon}{k} \min _{R}\left(\frac{Y_{R}}{v_{R, r}^{\prime} M_{w, R}}\right), \\
R_{i, r}=v_{i, r}^{\prime} M_{w, i} A B \rho \frac{\epsilon}{k} \frac{\sum_{P} Y_{R}}{\sum_{j}^{N} v_{R, r}^{\prime \prime} M_{w, j}},
\end{gathered}
$$

where $A=4.0$ - empirical constant; $B=0.5$ - empirical constant; $Y_{P}$ the mass fraction of product $P ; Y_{R}$ - the mass fraction of reagent $R ; N$ - number of reactions; $v_{i, r}^{\prime}$ - stoichiometric coefficient of reagent $i$ in reaction $r ; v_{i, r}^{\prime \prime}-$ stoichiometric coefficient of product $i$ in reaction $\mathrm{r} ; M_{w, i}$ - the molecular weight of species $i, \mathrm{~kg} / \mathrm{mol}$.

The chemical substance i formation rate is determined as the sum of the its formation rates according by Arrhenius law for each reaction:

$$
R_{i}=M_{w, i} \sum_{r=1}^{N_{R}} R_{i, r}
$$

The fuel reaction heat is determined in according by the known calorific value:

$$
\Delta H=\sum_{r=1}^{N} h_{i}^{0}\left(v_{i, r}^{\prime \prime}-v_{i, r}^{\prime}\right)
$$

The heat transfer equation is solved in order to determine the thermal state:

$$
\frac{\partial T}{\partial t}=a \nabla^{2} T+\frac{S_{h}}{c_{p} \cdot \rho},
$$

where $a=\lambda /\left(c_{p} \cdot \rho\right)$ - coefficient of thermal diffusivity, $\mathrm{m}^{2} / \mathrm{s}$. 
The specific heat capacity of each component does not depend on temperature. The specific heat capacity of the mixture is determined as the sum of the mass fractions of each component:

$$
c_{p}=\sum_{i} Y_{i} c_{p, i}
$$

The used boundary condition for the boundary G3 (Fig. 2) mean, that gas fuel flow enters through the pipeline dy 80 with defined mass flow and the velocity profile according by the turbulent regime: $\partial \mathrm{v} / \partial \mathrm{n}=$ const.

The boundary condition for the boundary G4 describes admission the flow of the watermethanol solution with defined mass flow rate: $\partial v / \partial n=$ const .

The boundary condition for the boundary G2 describes admission the flow of air with temperature equal to $0^{\circ} \mathrm{C}$.

The soil profile including the hill limits the burning area of the torch. This is described in according the boundary conditions G5. The lower boundary describes a wall, with the condition of adhesion, according by fallowing equations: $\partial T / \partial n=0 ; \partial c / \partial n=0$; $\partial v / \partial n=0$.

The boundary condition for the boundary G1 is described by soft boundary conditions.

\section{Results and discussion}

These differential equations are solved in the software module ANSYS Fluent in a twodimensional flat formulation. The results of the mathematical experiments are presented as a distribution of temperature and component composition (Fig. 2).

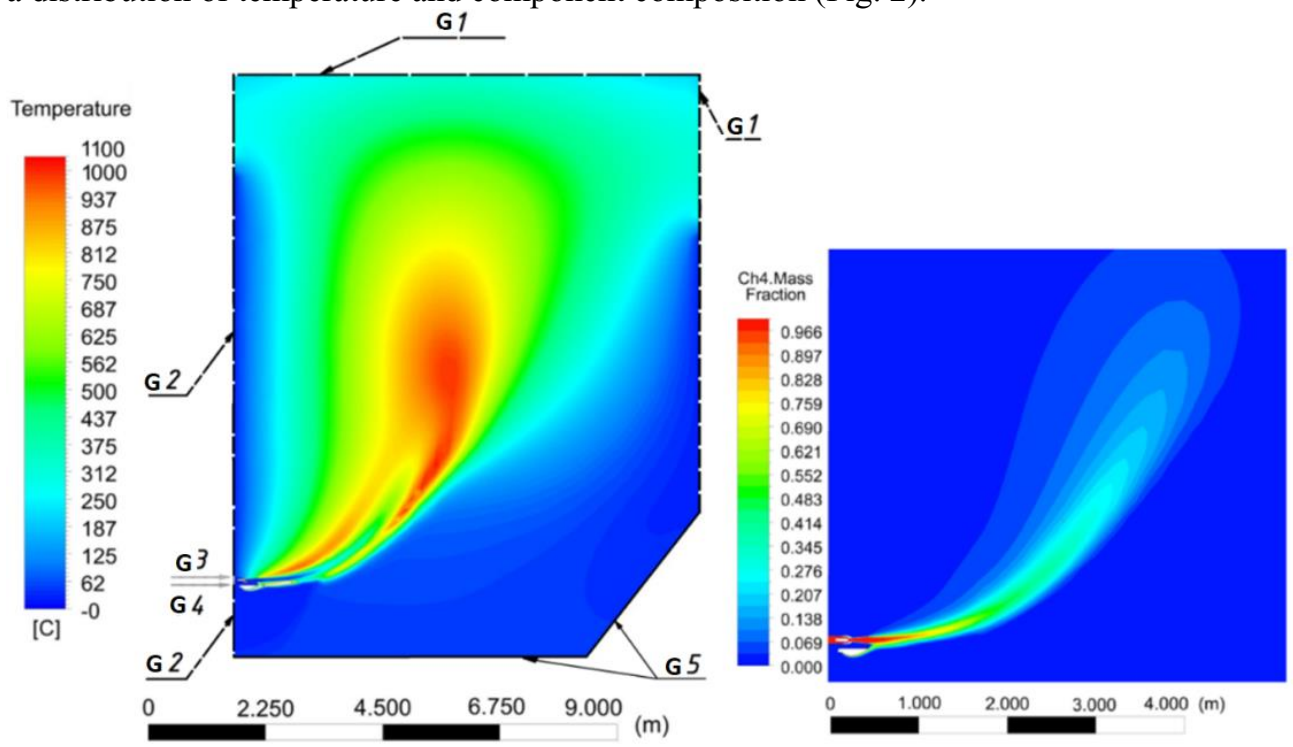

Fig. 2. Thermal distribution of the flare and distribution of methane concentration.

The facility is shown in Fig. 3 with flare geometric parameters when combusted defined mass flow the gas fuel and the water-methanol solution. 


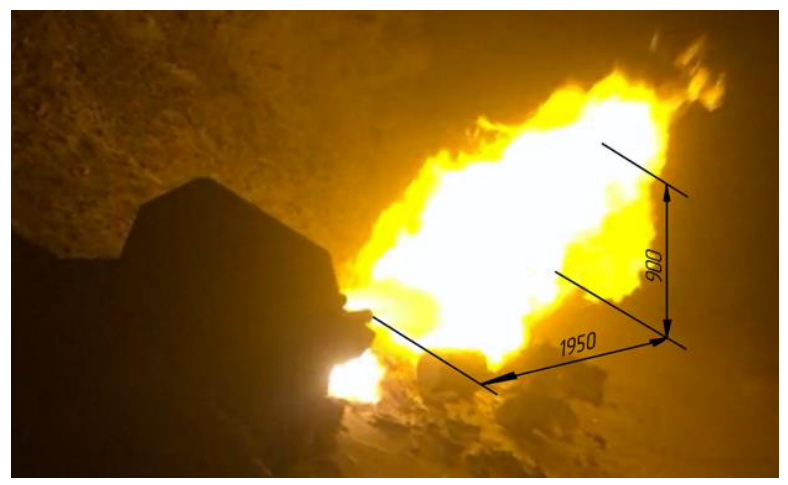

Fig. 3. The facility with flare geometric parameters.

The results of mathematical experiments satisfactorily correspond to the real dimensions of the facility flame. The formation of carcinogenic substances does not occur. The average temperature at the periphery of spraying the solution is about $1000{ }^{\circ} \mathrm{C}$. The mathematical model allows us to determine the heat flux in the necessary direction.

The mathematical model may be the basis for further study of waste disposal in burner facilities.

\section{References}

1. M. Vasilevsky, A. Razva, A. Alibek, B. Roman, MATEC Web Conf. 100, 01054 (2016)

2. N. A. Sozonov, Exposition Oil Gas 4, 90, (2014)

3. G. K. Batchelor, An Introduction to Fluid Dynamics (Cambridge Univ. Press, Cambridge, 1967)

4. H. J. Merk, Appl. Sci. Res. 8, 73, (1958)

5. B. E. Launder, D. B. Spalding. Lectures in Mathematical Models of Turbulence (Academic Press, London, 1972)

6. R. Taylor, R. Krishna, Multicomponent Mass Transfer (John Wiley and Sons, New York, 1993)

7. K. Y. Kuo, Principles of Combustion (John Wiley and Sons, New York, 1986)

8. T. H. Shih, W. W. Liou, A. Shabbir, Z. Yang, J. Zhu, Computers Fluids 24, 227 (1995)

9. B. F. Magnussen, B. H. Hjertager, On mathematical models of turbulent combustion with special emphasis on soot formation and combustion.In 16th Symp. on Combustion (The Combustion Institute, Pittsburgh, 1976) 